\title{
Tribune
}

\section{Il y a peut-être un avenir pour la pédagogie médicale...}

\author{
Charles $B O B$ 且 $\mathrm{N}^{\prime \prime}$
}

\section{Un avenir ! Quel avenir ?}

II arrive de dire de quelqu'un qu'il a un bel avenir devant lui quand on a confiance qu'il accédera un jour à des responsabilités lui permettant d'influencer le cours des événements. Q u'en est-il de la pédagogie médicale? $M$ on opinion est que son avenir dépendra essentielle ment de sa capacité à assumer efficacement la mission de « formation de médecins pour répondre aux besoins des citoyens » et de la reconnaissance de cette capacité par des instances concernées par l'avenir de la profession médicale: décideurs politiques, autorités sanitaires, organisations professionnelles, société civile et institutions académiques elles-mêmes.

Or, que peut-on constater aujourd'hui ? Comme le dieu romain Janus avec deux visages orientés dans des directions opposées, on peut soit observer un avenir sombre pour la pédagogie médicale, soit un avenir radieux. j'explique cette ambivalence, que j'ai moi-même vécue au cours de mon itinéraire professionnel, par un malentendu.

Reportons-nous au tableau 1 ci-après, qui présente la «mission » de la pédagogie médicale, déclinée en quatre composantes. Le malentendu réside dans le fait de penser que toute intervention de pédagogie médicale (composante $n^{\circ} 1$ dans la marge de gauche ou « pédagogie des cendante ») peut avoir une influence sur les autres composantes et conduire inéluctablement à une réponse aux besoins de santé des citoyens $\left(n^{\circ} 4\right)$. Ce postulat, quoiqu' implicite, est néanmoins largement répandu. La réalité est que l'effet de cascade ne s'opère pas nécessairement car les quatre composantes peuvent rester indépendantes les unes des autres. En effet, une intervention pédagogique, aussi innovante soit-elle pour rendre un apprentissage attractif et productif, ne peut être qu'une étape et un moyen pour atteindre une finalité sociale. Pour s'approcher de cette finalité, un accompagnement d'autres interventions est nécessaire.

Chacun peut convenir de cela, et néanmoins ne pas simpliquer dans une approche par système, qui permet de suivre l'effet de son intervention à travers un processus complexe. Ainsi un jour, un doyen de faculté de médecine d'un pays du $M$ oyen-O rient, féru de pédagogie médicale, me déclara avec une certaine fierté que son institution formait les meilleurs médecins de toute la région, en expliquant que ses jeunes médecins avaient les meilleurs scores à l'examen USLM E (U nited States Licencing M edical Examination). En conséquence de quoi, la moitié de ces médecins quittaient le pays pour sinstaller en Amérique du Nord. Ainsi donc, ce pays pauvre et sous-médicalisé, en particulier dans le milieu rural, perdait chaque année la moitié de ses effectifs médicaux après les avoir formé à grand frais pour les exporter vers un pays riche, et sans contrepartie.

C'est l'archétype d'une pédagogie médicale sans repère et sans avenir, celle à qui on ne reconnait pas la capacité d'influencer une situation de santé, ce que l'énoncé de sa mission laissait pourtant entendre (note: les expres sions " pédagogie médicale » et " éducation médicale » seront utilisés dans cet article pour signifier la même chose, bien que l'on puisse faire valoir une spécificité sémantique). L'horizon de cette pédagogie s'arrêteà l'enceinte de l'institution, avec peu d'impact sur la nature des effectifs médicaux (voir Tableau $1: n^{\circ} 2$ dans la marge de gauche), ni sur les conditions de pratique $\left(n^{\circ} 3\right)$, ni sur les besoins de santé de la population $\left(n^{\circ} 4\right)$.

Les effets d'une telle pédagogie peuvent être observés

${ }^{1}$ Consultant international en systèmes et personnels de santé - Ancien coordonnateur du programme del'Organisation Mondiale de la Santé (OMS) des ressources humaines pour la santé - Membre du conseil d'administration de la SIF̈M (Société Internationale Francophone d'Education Médicale) - mailto:boelen.charles@wanadoo.fr.

* Texte élaboré à partir d'une conférence présentée au Congrès IPM 2004 (Internet et Pédagogie médicale) - Grenoble France 2 décembre 2004. 


\begin{tabular}{|ccc|}
\hline \multicolumn{3}{|c|}{$\begin{array}{c}\text { Tableau } 1 \\
\text { Pédagogie médicale « descendante } \\
\text { ou pédagogie médicale « montante }\end{array}$} \\
\hline 1 & Formation & 4 \\
2 & de médecins & 3 \\
3 & pour répondre & 2 \\
4 & aux besoins des citoyens & 1 \\
\hline
\end{tabular}

dans de nombreux pays, nantis comme émergents. Il faut reconnaître que nos pères fondateurs, $\mathrm{H}$ ippocrate pour la pratique médicale et Abraham Flexner pour l'éducation médicale, ne nous ont guère habitué à l'approche globale. En effet, le serment d'H ippocrate reste très en retrait par rapport à la position d'un médecin agissant sur les déterminants de la santé, pour se limiter aux principes du colloque singulier. Q uant à Flexner, à qui il faut quand même reconnaître l'émergence d'une fondation scientifique à la formation médicale, on lui doit le legs universel d'une dichotomisation du programme des études médicales, avec une charge souvent excessive de sciences dites de base en premières années de formation et la consolidation d'un schisme avec les réalités du terrain et du contexte social. Son paradigme est devenu aujourd'hui obsolète ${ }^{1}$.

Par contre, une pédagogie d'avenir effectue une démarche inverse (voir Tableau 1 : la marge de droite ou " pédagogie montante »), en s'inspirant d'abord des besoins des citoyens $\left(n^{\circ} 1\right)$, en s'interrogeant sur la capacité du système de santé à y répondre $\left(n^{\circ} 2\right)$ et en se réfé rant à un profil de médecin $\left(n^{\circ} 3\right)$ susceptible de fonctionner au mieux dans ce contexte. Cette démarche sapplique à analyser ce qui peut sembler aller de soi et à rendre toute situation explicite.

- Ainsi, s'inspirer des « besoins des citoyens » revient à identifier un socle de valeurs appelé à conditionner la pédagogie médicale: qualité, équité, pertinence et coûtefficacité du service de santé.

- S'interroger sur la manière de « répondre » aux besoins des citoyens amène à comprendre le fonctionnement d'un système de santé, ses forces, ses faiblesses et les réformes nécessaires pour adapter le cadre professionnel dans lequel les futurs médecins seront amenés à fonctionner.

- Enfin, définir clairement le rôle d'un médecin, c'est identifier le champ de compétences nouvelles que le contexte socio-économique et culturel attend de lui et autour desquelles le programme de formation et la pédagogie médicale seront construits.

\section{Q uels besoins? Q uel système de santé?}

C ertes, ce n'est pas à la faculté de médecine, à travers la pédagogie médicale, d'apporter principalement des réponses à ces questions, mais aux experts dans ces domaines. N éanmoins, la faculté de médecine toute entière doit se sentir solidairement impliquée car, comme on peut l'attendre de toute institution d'intérêt public, elle doit être soucieuse du devenir de son «produit » et de son impact sur la société, et la pédagogie médicale tout autant.

Reconnaissons-le, la pédagogie « descendante » n'a pas cette culture.

Q uant à la pédagogie «montante », pour s'épanouir pleinement et durablement, elle devra être hébergée dans une faculté capable d'anticipation et d'ouverture sur le monde extérieur.

Comme évoqué par la poignée de mains tendue par I'institution académique (Figure 1), on peut caractériser «l'ouverture » par trois critères:

- une écoute du monde extérieur, exemplifié par l'appréciation des défis actuels et potentiels dans la société, l'identification de problèmes prévalents de santé et des populations plus vulnérables, I'adaptation des fonctions de l'institution, dont la fonction de formation, à ces défis et réalités.

- une collaboration avec les principaux acteurs du secteur de la santé pour mieux assumer sa mission, tels que: instances politiques, organisations de service de santé, associations professionnelles, société civile, et autres institutions académiques.

- un engagement à améliorer le fonctionnement du système de santé dans lequel vont être affectés les futurs médecins afin qu'ils trouvent un milieu propice à pouvoir exercer les compétences acquises au cours de leur formation.

Cette ouverture peut s'exprimer concrètement de diffé rentes manières suivant le milieu. En tout cas, elle illustre la volonté de l'institution d'être considérée 


\section{Tribune}

comme un acteur important sur l'échiquier de la santé, autant pour l'analyse du système de santé que pour la recherche de stratégies d'action. La faculté, tenante d'une pédagogie «montante», c'est-à-dire guidée par la finalité de son action, peut tenir cette posture. A défaut, elle risque d'être marginalisée quand viendra le temps de prendre des décisions susceptibles d'avoir des incidences importantes sur le système de santé, y compris sur son propre avenir. Ainsi, ai-je été étonné, à la lecture du récent rapport du $\mathrm{H}$ aut Conseil sur l'Avenir de I'Assurance-M aladie, en France, de ne pas voir figurer la faculté de médecine parmi ses membres, alors qu'ont été consultés: élus, représentants des assurés sociaux, des employeurs, des régimes d'assurance maladie, de l'E tat, des professions de santé, des établi ssements de santé, des usagers ${ }^{2}$. Faut-il se résoudre à croire que l'avis de la faculté de médecine n'a pas été jugé indispensable pour formuler des recommandations pour l'amélioration du fonctionnement du système de santé et de l'offre médicale, malgré les incidences importantes pour la réorientation de la formation médicale?

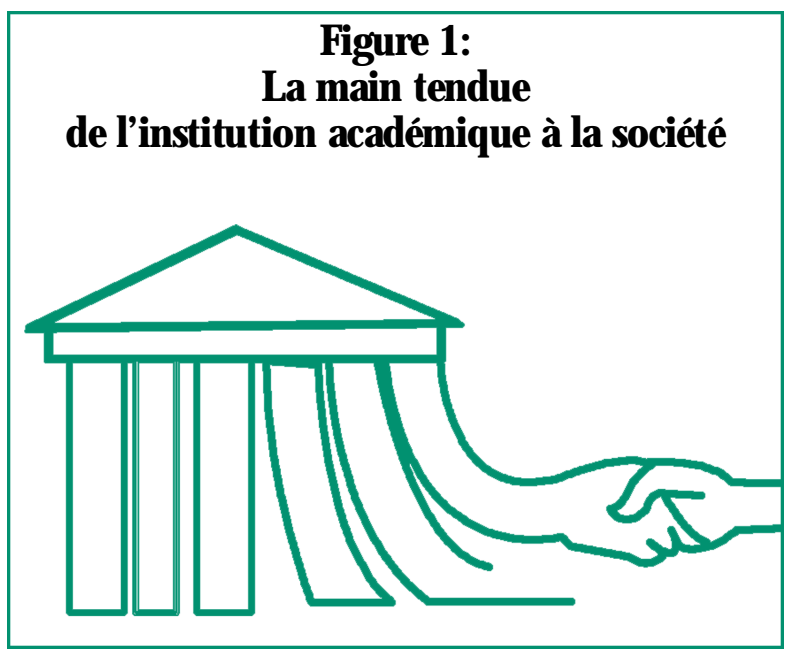

Cet événement ne peut cependant occulter les efforts de certaines facultés françaises à devenir des interlocuteurs écoutés dans le débat social. La faculté de médecine et de pharmacie de Besançon, par exemple, s'est distinguée par un colloque tenu en 2002 autour de la question: "Quelle responsabilité sociale pour les facultés de médecine? ", et auquel participaient les principaux partenaires de santé de la région et du pays ${ }^{3}$. Cette ques tion n'était pas innocente puisqu'elle incitait les autres partenaires à se poser la même question et à faire le constat de la nécessité d'une coordination entre politiques de santé et institutions académiques, suivant en cela l'approche développée par l'OM S, « Vers l'unité pour la santé $»^{4}$.

C ette approche fait état, en effet, d'une fragmentation du système de santé, due en partie à des missions incohérentes, en tout cas peu coordonnées, que se sont fixées décideurs politiques, gestionnaires de la santé, professions de santé, institutions académiques et société civile, entraînant des conflits entre intérêts particuliers et intérêt public. Par ailleurs, elle invite ces mêmes acteurs du système de santé à réajuster leurs missions en fonction d'un même socle de valeurs (voir le centre du pentagone dans la Figure 2), à réexaminer leurs actions pour mieux y répondre et à établir entre eux des partenariats. Une telle approche permet à la faculté de médecine de mieux comprendre son propre positionnement dans la complexité du système de santé et à opter pour une stratégie assurant un meilleur impact de ses actions.

L'avenir de la pédagogie médicale tient à un tel engagement.

\section{Quels médecins?}

On est étonné en consultant les prospectus des facultés de médecine de n'y trouver que très rarement une description claire du produit qu'elles s'engagent à former, comme s'il n'échappait à personne ce que « bon médecin » veut dire. Se réfugier dans l'implicite serait-il un vestige d'arrogance d'un autre âge, alors que la société civile appelle aujourd'hui à l'explicite et à la transparence pour faire valoir démocratiquement ses droits et exprimer ses griefs ? II est impératif de dire intelligiblement ce que l'on attend d'un médecin pour répondre aux besoins des citoyens et aux exigences des futurs systèmes de santé.

Ceci dit, il est rassurant de constater que cet exercice de prospective trouve la faveur auprès de certaines organisations. Ainsi, le ministère marocain de la santé tiendra une conférence de consensus en 2005 pour définir le profil de poste du futur médecin généraliste, en pleine concertation avec les institutions concernées, dont les facultés de médecine.

Par ailleurs, en 2004, la fondation américaine de médecine interne et la fédération européenne de méde cine interne publièrent une plaquette, "T he Physician Charter», qui décline trois principes de base autour des- 
quels le nouveau rôle du médecin devrait être défini : bien-être du patient, autonomie du patient et justice sociale ${ }^{5}$. Le principe de justice sociale est ainsi formulé qu'on attend de la profession médicale qu'elle adapte sa pratique, en plus de la traditionnelle exigence du colloque singulier, aux nouveaux défis de société et à un usage plus équitable des ressources de santé.

Enfin, lors de son dernier congrès mondial (octobre 2004), I'O rganisation M ondiale des M édecins de Famille (W O N CA) a jugé bon de décerner un prix à un médecin répondant au mieux au profil du médecin «cinq étoiles », défini par l'OM S en 1994 comme le prototype du médecin de l'avenir ${ }^{6}$. Un tel trophée sera ainsi remis à chacun de ces congrès.

De la faculté de médecine, on ne pourra pas attendre moins. Elle devra rendre plus explicite son projet de formation. La pédagogie d'avenir sera celle qui inscrira au frontispice de chaque faculté de médecine le type de médecin qu'elle s'engageà former en justifiant son choix en fonction des réalités du contexte et de l'évolution du système de santé. Elle se portera garante du fait que la conception de toute intervention pédagogique s'inspirera de ce modèle et des aptitudes essentielles qu'il promeut, tant pour le contenu de l'enseignement, les modalités d'apprentissage et l'évaluation des acquis. La pédagogie de l'avenir ne se réduira pas à un bureau technique de la faculté, appliquant les dernières technologies de communication à l'apprentissage ou soutenant les enseignants et apprenants dans l'organisation de I'enseignement ou de l'évaluation. Elle sera un centre d'excellence qui aidera la faculté de médecine à avoir une meilleure maîtrise sur ses produits et à assurer leur intégration optimale dans le contexte sanitaire et social.

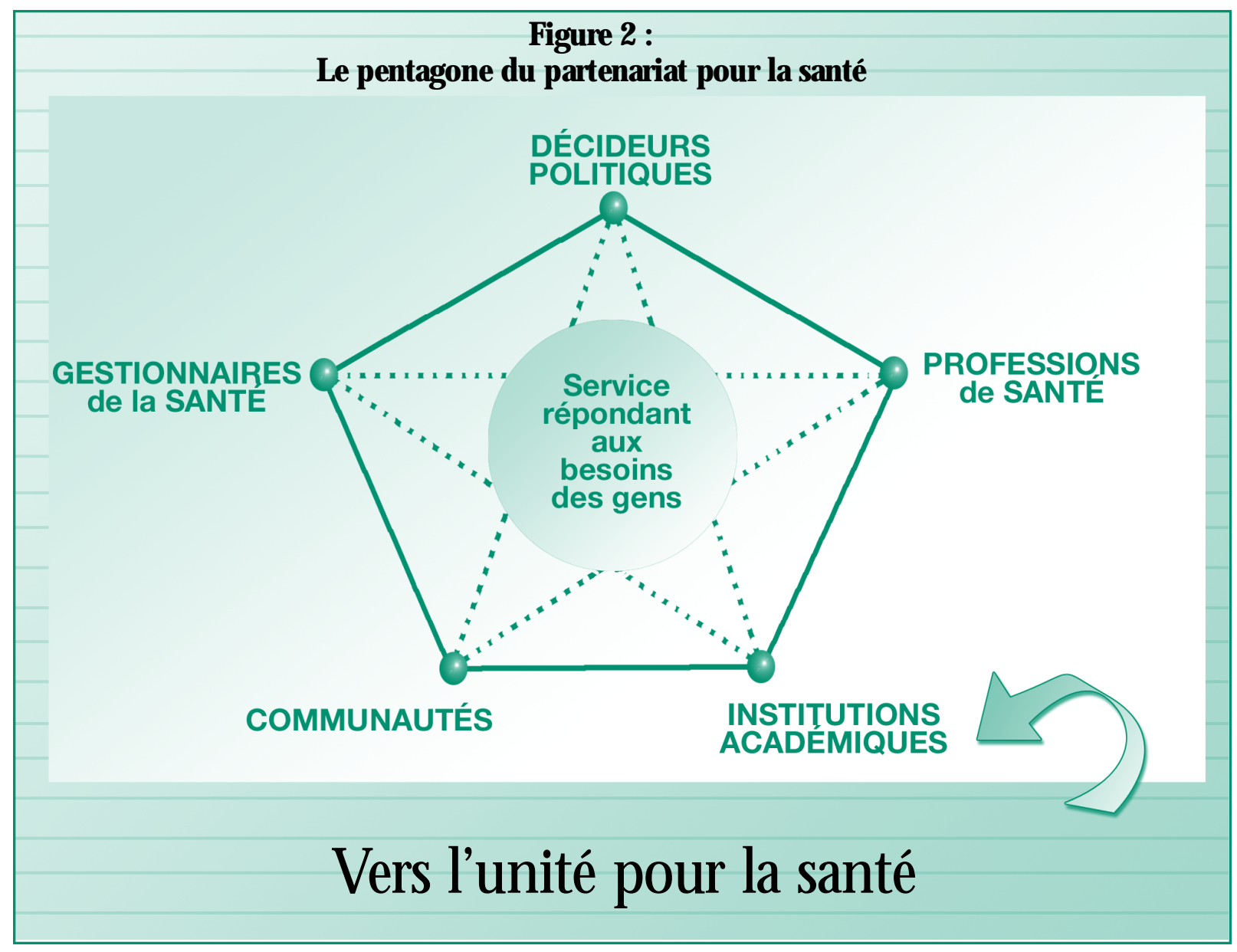




\section{Tribune}

\section{Une culture de résultats}

La faculté de médecine, comme toute autre institution, sera de plus en plus soumise à une culture de résultats. En cela elle sera comptable devant la société, sachant qu'elle ne sera pas seule à décider des résultats attendus $\mathrm{d}^{\prime} \mathrm{elle}^{7,8}$. Ainsi, en matière de formation, sa performance pourrait être déclinée, entre autres critères, en fonction du nombre de médecins formés répondant aux critères d'un modèle publiquement annoncé, de l'encouragement au choix de carrières et de sites d'installation en fonction des besoins du public, de l'offre d'un méca nisme de formation continue, et de la contribution à la préparation de meilleures conditions de pratique, par la négociation et la recherche.

Certes, la mission de formation sera d'autant plus cohé rente que le positionnement de la faculté de médecine sera volontariste vis-à-vis d'une évolution du système de santé. Cette culture de résultats devrait affecter également les missions de recherche et de dispensation de services de santé, et être reflétée dans des normes d'accréditation.

Faut-il s'étonner de cette évolution? Pas vraiment, quand on fait un retour sur l'histoire de la pédagogie ou de l'éducation médicale (voir Figure 3).

Reconnaissons que l'éducation médicale moderne est née au lendemain de la seconde guerre mondiale, appliquant les théories du management d'abord utilisées par les militaires et les industriels. Commence alors ce qu'on peut appeler la période du " processus », caractérisée par un souci de mieux gérer l'éducation médicale en appliquant la planification par objectifs, la mise en opérations et l'évaluation. Cette approche gagne en popularité pour arriver à un plateau dans les années soixantedix et continue à servir de norme pour une bonne gouvernance de toute intervention pédagogique. D es progrès continuent à être observés concernant les modalités d'apprentissage et d'évaluation des performances des étudiants, notamment par l'application de technologies diverses.

Vient ensuite une autre vague qu'on appellera celle de la "pertinence », émergeant avec le questionnement sur le rôle des institutions et la critique de mieux servir l'intérêt public. On s'interroge sur la finalité de la technologie éducative et sur sa cohérence avec l'évolution sociale. $D$ ans les pays nouvellement indépendants, on tente de réajuster la pédagogie médicale héritée de la puissance coloniale pour l'adapter aux conditions pré valentes dans les pays et les communautés locales. $D$ ans les pays industrialisés, le mouvement contestataire étudiant contribue égal ement à l'émergence de cette vague. D'autre part, I'OMS, s'appuyant sur sa politique de « santé pour tous » et sa stratégie des soins de santé primaires, encourage cette mise en cohérence entre formation et besoins des communautés.

Enfin, apparaît au début des années quatre-vingt-dix la vague de l' « impact ». Avec le phénomène de globalisation, l'éducation médicale se voit progressivement soumise aux mêmes règles que toute autre activité humaine: savoir-faire dans la gestion de systèmes complexes, recherche de performance, traçabilité des résultats, compétitivité, transparence et évaluation.

D es sujets, jusque là considérés comme quasiment profanatoires, animent progressivement des débats publics: relativité des contributions au progrès social à mettre au crédit de la formation et de la recherche médicales, réexamen des missions des institutions académiques et publication du résultat de leur actions, impact discutable de la pédagogie médicale sur le bien-être des populations en l'absence d'uneinfluence sur l'environnement de travail des futurs gradués pour pratiquer les aptitudes acquises à la faculté.

On aura compris que la critique n'est pas restreinte à I'application d'un corpus de connaissances bio-médicales, mais qu'elle s'applique surtout à la capacité à servir les valeurs de qualité, d'équité, de pertinence et de coût-efficacité dans la prestation des soins de santé.

\section{Vers un nouveau paradigme}

Chaque nouvelle vague est un enrichissement de la précédente. II nous semble que la pédagogie de l'avenir est celle qui saura répondre aux exigences de la nouvelle vague de l'impact, tout en utilisant les acquis des vagues précédentes.

Au C anada, pays pionnier en matière d'innovations en éducation médicale, l'association des facultés de médecine canadiennes confirme cette tendance dans sa plaquette élaborée avec le soutien du gouvernement fédé ral : «Imputabilité sociale. U ne vision pour les facultés de médecine du $C$ anada $»$.

On peut s'attendre, à terme, à ce que les gouvernements, agences internationales et fondations diverses ne soutiennent activement que cette forme de pédagogie, capable de se fixer comme ligne d'horizon la finalité de 


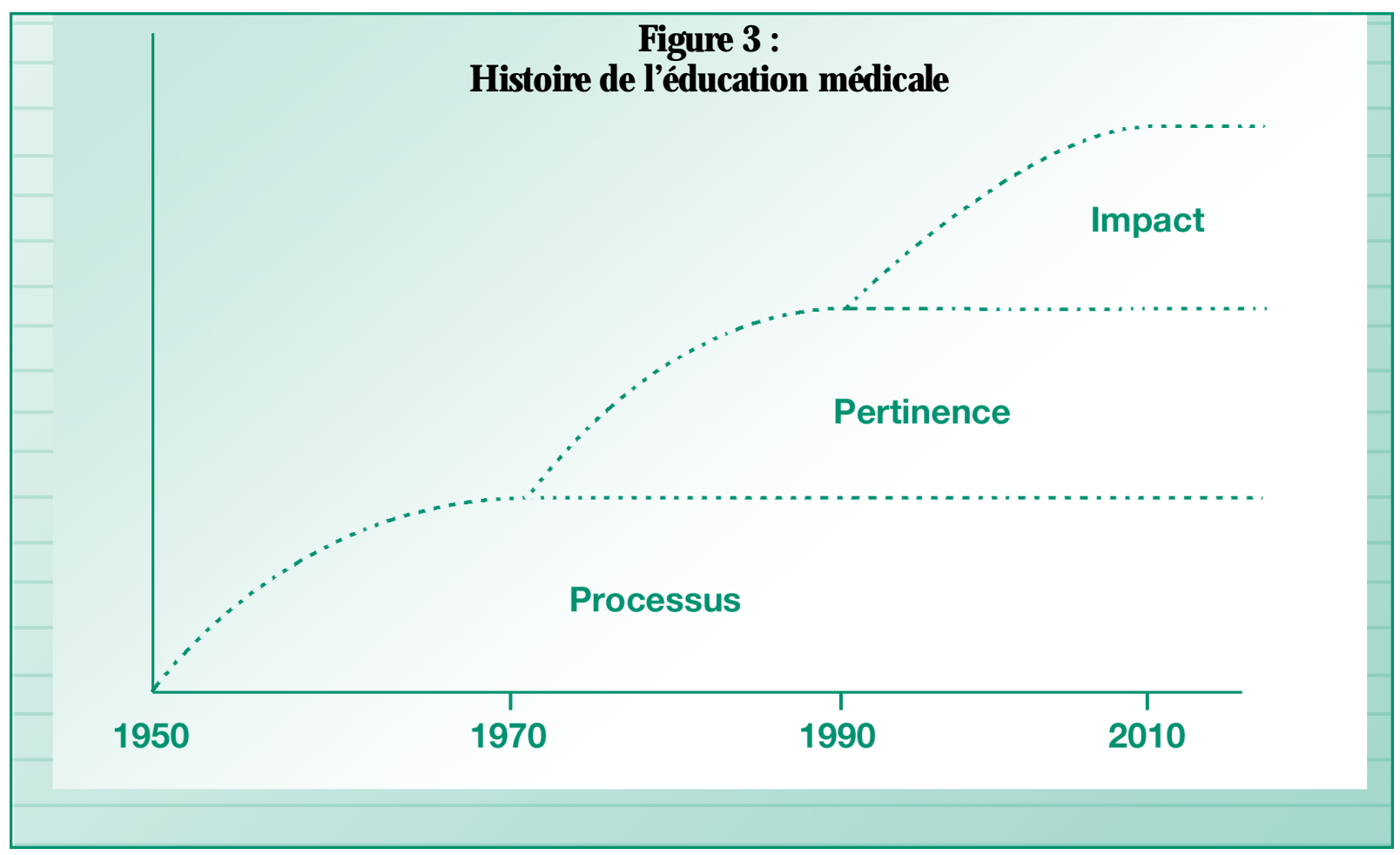

son action et intimement associée au devenir de la profession médicaleet du système de santé. D e plus, les facultés de médecine elles-mêmes, comprenant mieux leur potentiel et leur responsabilité dans le développement sanitaire, nemanqueront pas d'investir préférentiellement dans ce type de pédagogie, militante et investigatrice ${ }^{10}$.

Certes, pour les promoteurs de cette pédagogie d'avenir, les actions concrètes pourront être multiples. Elles nepeuvent être traitées dans ce court article. Cependant, on peut décliner quelques principes généraux assortis d'exemples, conformes à cette pédagogie:

- immersion dans la réalité. Par exemple, proposer à un étudiant de suivre une même famille pendant toute sa formation afin qu'il apprenne à comprendre et maîtriser les événements de santé survenant dans ce milieu de proximité.

- interrogation sur la finalité. Par exemple, guider l'étudiant dans son choix de carrière, éventuellement vers la médecine générale où les besoins sont les plus grands, et participer aux délibérations sur la place nouvelle de cette discipline dans le futur système de santé.

- action sur le système de santé Par exemple, initier ou participer à un programme relatif aux maladies chroniques pour une population en faisant intervenir de façon coordonnée les principaux acteurs du système de santé, avec lesquels la faculté désire établir un partenariat. - promotion de la transparence. Par exemple, développer des mécanismes d'évaluation de la performance et de l'impact de la faculté de médecine sur le développement sanitaire, et promouvoir un mécanisme d'accréditation tenant compte de ces données.

\section{Conclusion}

Y a-t-il un avenir pour la pédagogie médicale?... Sûrement! 


\section{Tribune}

\section{Références}

1. Boelen C. A new paradigm for medical schools a century after Flexners report. Bulletin of the World $\mathrm{Health}$ O rganization $2002 ; 80: 592-593$

2. Rapport du H aut conseil pour l'avenir del'assurancemaladie, Paris, France, 23 janvier 2004. [On-line] disponible sur: http://www. assurancemaladiesante gouv.fr/pdf/rapport.pdf

3. Quelle responsabilité sociale pour les facultés de médecine? Santé Publique, revue de la Sociéé Française de Santé Publique, N uméro H ors série, avril 2003.

4. OM S. Vers I'U nité pour la santé. Défis et opportunités de partenariats pour ledéveloppement dela santé $D$ ocument de travail, Genève, Suisse, 2002 (WH O/EI P/O SD /2000.9).

5. ABIM Foundation, ACP Foundation, European Federation of Internal M edicine M edical professionalism in the new millenium. A Physician Charter, Ann Intern M ed $2002 ; 163$ : 243-246. Traduction française: Le profes sonnalisme médical pour le nouveau millénaire: une charte pour les praticiens. Pédagogie M édicale 2004 ; 5 : 43-45.
6. OM S. M édeains pour la santé U ne stratégie mondiale de I'OM S pour la réorientation de l'enseignement de la médecine et de pratique médicale en faveur de la santé pour tous, Genève, Suisse, 1996 (WH O/H RH/96.1).

7. Boden $C, H$ eck J. D éinir et mesurer la responsabilité sociale des facultés de médecine, OMS, Genève, Suisse, 2000 (WH O/HRH/95.7).

8. Barrier J, Roland J (éditeurs). La facultédemédeanefaceà ses responsabilités sociales et éhiques. CID M EF en collaboration avec I'O M S, 2004.

9. Santé Canada. Imputabilité sociale U ne vision pour les facultés de médecine du Canada, Ottawa $(\mathrm{ON})$ : Publications Santé Canada, 2001. [0 n-line] disponible sur : http://www.hc-sc.gc.ca/hppb/soinsdesante/pubs/ imputabilite_socialel

10. Boelen C. Health challenges and medical schools. Forum bulletin de l'Association des Facultés de M édecine du Canada 2004; 37: 1-6. N ${ }^{\circ} 1$, mars 2004. [On-line] disponible sur: http://www.afmc.ca/pages/fr_publications_forum.html 